\title{
Satellites of massive galaxies: the infalling pieces of the puzzle
}

\author{
E. Mármol-Queraltó ${ }^{1,2}$, I. Trujillo ${ }^{1,2}$, P. G. Pérez-González ${ }^{3}$, \\ G. Barro ${ }^{4}$, J. Varela ${ }^{5}$, and V. Villar ${ }^{3}$ \\ ${ }^{1}$ Instituto de Astrofísica de Canarias, c/Vía Láctea s/n, E-38205, La Laguna, Spain \\ ${ }^{2}$ Dpto. de Astrofísica, Universidad de La Laguna, E-38205, La Laguna, Spain \\ ${ }^{3}$ Dpto. de Astrofísica, CC. Físicas, Universidad Complutense de Madrid, E-28040, Spain \\ ${ }^{4}$ UCO/Lick Observatory, University of California, Santa Cruz, CA 95064 \\ ${ }^{5}$ Centro de Estudios de Física del Cosmos, Plaza de San Juan, E-44001, Teruel, Spain
}

\begin{abstract}
Accretion of minor satellites has been postulated as the most likely mechanism to explain the significant size evolution of massive galaxies over cosmic time. A direct way of probing this scenario is to measure the frequency of satellites around massive galaxies at different redshifts. Here we present our study of satellites around massive galaxies $\left(M_{\text {star }} \sim 10^{11} \mathrm{M}_{\odot}\right)$ up to $z \sim 2$. We find (Fig. 1) that the fraction of massive galaxies with satellites down to 1:10 mass ratio is $\sim 15 \%$ ( $\sim 30 \%$ down to 1:100), not varying with redshift (Mármol-Queraltó et al. (2012)). We also find that our satellites are younger than their central galaxies at low z (Mármol-Queraltó et al., 2013). Then, if minor merging is acting to form massive galaxies, their ourtskirts should be younger than their cores. The challenge to find this age gradient in nearby massive galaxies is opened.
\end{abstract}

Keywords. galaxies: massive galaxies, galaxies: evolution galaxies: high-redshift galaxies:formation

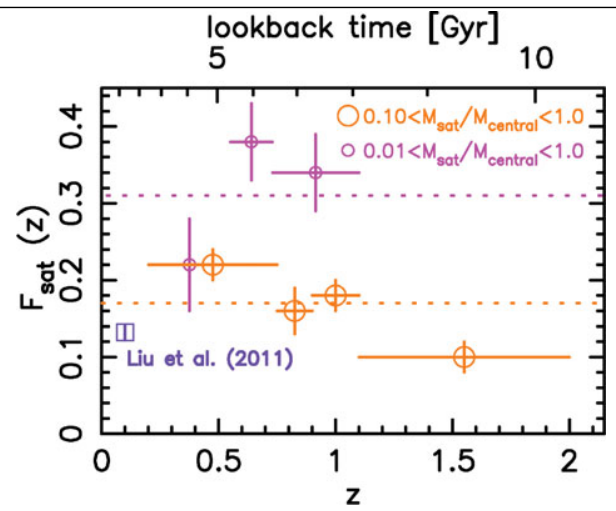

Figure 1. Fraction of massive galaxies with satellites within a projected distance of $100 \mathrm{kpc}$ for different redshifts. The magenta small circles indicate the fraction of massive galaxies with satellites down to 1:100 mass ratio, and the orange big circles down to 1:10 mass ratio. The blue cross indicates the fraction of satellites around massive galaxies in the nearby universe $(\sim 12 \%$, Liu et al. (2011)), in agreement with our results at higher redshifts.

\section{References}

Liu, L., Gerke, B. F., et al. 2011, ApJ, 733, 62

Mármol-Queraltó, E., Trujillo, I., Pérez-González, P., Varela, J., \& Barro, G. 2012, MNRAS, 422,2187

Mármol-Queraltó, E., Trujillo, I., Villar, V., Barro, G., \& Pérez-González, P. 2013, MNRAS, 429, 792 